\title{
Evaluation of inclusive breakup cross sections in reactions induced by weakly-bound nuclei within a three-body model
}

\author{
Jin Lei, Antonio M. Moro
}

Departamento de FAMN, Universidad de Sevilla, Apartado 1065, 41080 Sevilla, Spain

\begin{abstract}
In 1980s Ichimura, Austern and Vincent [Phys.Rev.C 32, 431 (1985)] proposed a quantum mechanical theory to study the problem of inclusive breakup cross sections in the nuclear reactions. Using the DWBA version of this model, applications to deuteron and ${ }^{6} \mathrm{Li}$ inclusive breakup reactions are presented and compared with some available data.
\end{abstract}

\section{Introduction}

The breakup of a nucleus into two fragments is an important mechanism in nuclear reactions, especially when one of the colliding nuclei is weakly bound. For example, $a+A \rightarrow b+x+A$, where $a=b+x$. If both fragments ( $b$ and $x$ ) are detected, the reaction is said to be exclusive. In addition, when all the fragments and target are emitted in their ground state, it is called Elastic Breakup (EBU). On the other hand, if only one of the fragments $($ say, $b)$ is detected, the reaction is inclusive. This includes the EBU, and also the target $(A)$ excitation, the fusion of $x$ with $A$, and the exchange of particles between $x$ and $A$, which are globally referred to as Nonelastic Breakup (NEB). 
The problem of calculating inclusive breakup cross sections was studied in 1980s by Pampus et al. [1], with the works of Udagawa and Tamura [2] and Austern and Vincent [3] appearing later. In [1-3], the authors considered the reactions in which the projectile is broken up into two fragments. These processes can be represented as $a+A \rightarrow b+B^{*}$, where $B^{*}$ is any possible state of the $x+A$ system. This includes the elastic scattering of $x+A$, which corresponds to the EBU, and non-elastic $x+A$ scattering, which gives rise to the NEB processes defined above. In [2], Udagawa and Tamura described this problem in the prior form DWBA formalism, whereas Austern and Vincent used the post form [3]. The later was refined by Kasano and Ichimura [4], who found a formal separation between EBU and NEB contributions. These results were carefully reviewed by Ichimura, Austern and Vincent [5] and the model was subsequently referred to as IAV formalism.

In this contribution, we have applied the finite-range DWBA version of IAV formalism $[5,6]$ to different inclusive breakup reaction systems and compared with the available data.

\section{Theoretical formulation}

Following Ichimura, Asutern and Vincent [5]. We write the process under study as,

$$
a(=b+x)+A \rightarrow b+B^{*},
$$

in which a projectile $a$ colliding with a target $A$ breaks into two fragments, $b$ and $x$. We assume that only $b$ is observed in the experiment and therefore the corresponding experimental cross section will correspond to a sum over all possible final states of the $x+A$ system. This process will be described with the Hamiltonian

$$
H=K+V_{b x}+U_{b A}\left(\vec{r}_{b A}\right)+H_{A}(\xi)+V_{x A}\left(\xi, \vec{r}_{x}\right),
$$

where $K$ is the total kinetic energy operator, $V_{b x}$ is the interaction between the clusters $b$ and $x$ in the projectile $a, H_{A}(\xi)$ is the Hamiltonian of the target nucleus (with $\xi$ denoting its internal coordinates) and $V_{x A}$ and $U_{b A}$ are the fragment-target interactions.

Note that we make a distinction between the two cluster constituents; the interaction of the fragment $b$, the one that is assumed to be detected in the experiment, is described with an optical potential. Non-elastic processes arising from this interaction (e.g. target excitation), are included through $U_{b A}$. The particle $b$ is said to act as spectator. On the other hand, the 
interaction of the particle $x$ with the target retains the dependence of the target degrees of freedom $(\xi)$.

Using the post form DWBA, the inclusive breakup differential cross section, as a function of the angle and energy of the fragment $b$, is given by

$$
\frac{d^{2} \sigma}{d E_{b} d \Omega_{b}}=\frac{2 \pi}{\hbar v_{a}} \rho\left(E_{b}\right) \sum_{c}\left|\left\langle\chi_{b}^{(-)} \Psi_{x A}^{c}\left|V_{\text {post }}\right| \chi_{a}^{(+)} \phi_{a}\right\rangle\right|^{2} \delta\left(E-E_{b}-E^{c}\right)
$$

where $V_{\text {post }}=V_{b x}+U_{b A}-U_{b B}$ and $c$ labels the states of $x$ - $A$ system. In the theory of IAV, they used Sokhotski-Plemelj theorem [7] and the Feshbach optical reduction, leading to the NEB differential cross section:

$$
\left.\frac{d^{2} \sigma}{d E_{b} d \Omega_{b}}\right|_{\mathrm{NEB}}=-\frac{2}{\hbar v_{i}} \rho_{b}\left(E_{b}\right)\left\langle\psi_{x}\left|W_{x}\right| \psi_{x}\right\rangle
$$

where $\rho_{b}\left(E_{b}\right)=k_{b} \mu_{b} /\left((2 \pi)^{3} \hbar^{2}\right)$ is the density of states for the particle $b$, $W_{x}$ is the imaginary part of the optical potential $U_{x}$ which describes $x+A$ elastic scattering. The function $\psi_{x}$, which represents the $x-A$ relative motion when the target in the ground state, verifies the equation

$$
\left(E_{x}^{+}-K_{x}-U_{x}\right) \psi_{x}=\left(\chi_{b}^{(-)}\left|V_{\text {post }}\right| \chi_{a} \phi_{a}\right\rangle,
$$

where $E_{x}=E-E_{b}, \chi_{b}^{(-)}$is the distorted-wave describing the scattering of $b$ in the final channel with respect to $B^{*}$.

\section{Calculations}

In this section, we present calculations for reactions induced by deuteron and ${ }^{6} \mathrm{Li}$ projectiles, and compare with the available experimental data. In all cases, we compute the separate contributions for the elastic (EBU) and non-elastic (NEB) breakup cross sections. For the former, we use the CDCC formalism [8], using the coupled-channel code FRESCO [9]. For the NEB part, we use Eq. (4). The intrinsic spins are ignored in the NEB Calculations.

First we consider the $d+{ }^{118} \mathrm{Sn}$ reaction at $E_{d}=56 \mathrm{MeV}$ and compare with the data from Ref. [10]. These data were already analyzed in Ref. [11], using the zero-range version of the post-form DWBA formula.

In the CDCC calculations the deuteron breakup is treated as inelastic excitations to the $p$ - $n$ continuum. These $p$ - $n$ continuum states are truncated in excitation energy and discretized in energy bins. For the $p-n$ interaction, we consider the simple Gaussian form of Ref. [8]. The proton-target and neutron-target interactions are adopted from the global parametrization of 

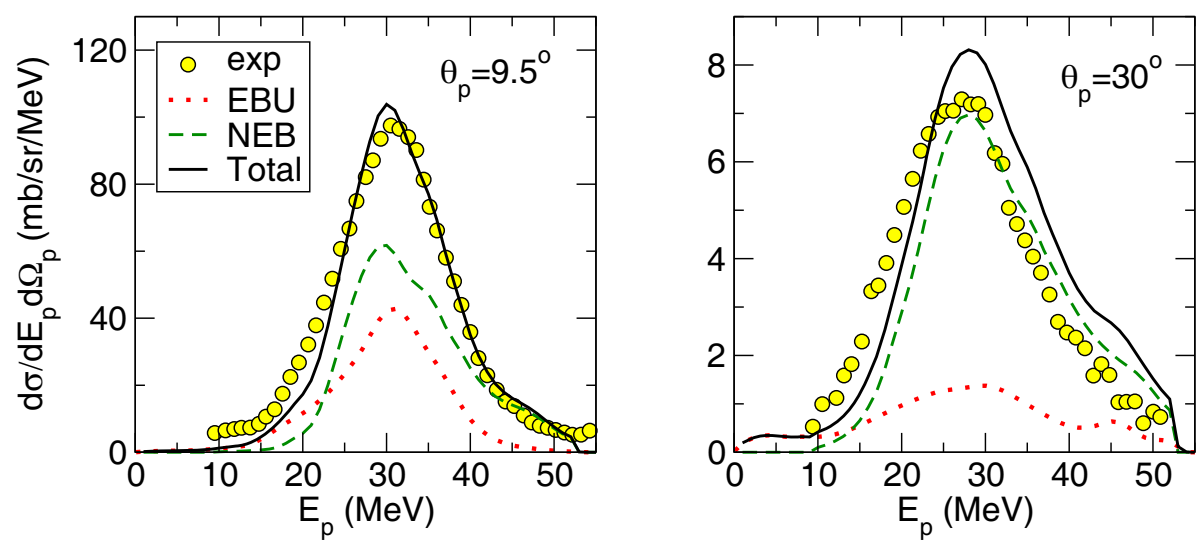

Figure 1: Experimental and calculated double differential cross section, as a function of the outgoing proton energy, for the protons emitted in the ${ }^{118} \mathrm{Sn}(d, p \mathrm{X})$ reaction with angles of $9.5^{\circ}$ (left panel) and $30^{\circ}$ (right panel) at incident energy of $56 \mathrm{MeV}$.

Koning and Delaroche (KD) [12], omitting the spin-orbit term, and evaluated at half of the deuteron incident energy. For the NEB calculations, we use also the KD parametrization for the proton-target and neutron-target interactions, but evaluated at the corresponding proton $\left(E_{p}\right)$ and neutron $\left(E_{n}\right)$ energies. In DWBA, one needs also the incoming channel optical potential $\left(d+{ }^{118} \mathrm{Sn}\right)$, which is taken from Ref. [13].

In Fig. 1 we compare the experimental and calculated inclusive double differential cross section, $\mathrm{d}^{2} \sigma / \mathrm{d} E_{p} \mathrm{~d} \Omega_{p}$, corresponding to different proton angles as a function of outgoing proton energy in the laboratory frame. The dotted line is the EBU calculation, which is found to underestimate the data. The dashed line is the calculation for the NEB part. The solid line is the sum of the EBU and NEB contributions. It is seen that for the smaller angle $\left(\theta_{p}=9.5^{\circ}\right)$, the contribution of EBU is comparable with NEB, whereas for the larger angle $\left(\theta_{p}=30^{\circ}\right)$, the inclusive breakup cross section is largely dominated by the NEB contribution. The summed EBU and NEB contributions reproduce reassembly well the magnitude and shape of the data.

As a second example, we calculate the inclusive $\alpha$ cross sections for the reaction ${ }^{6} \mathrm{Li}+{ }^{159} \mathrm{~Tb}$ at bombarding energies of $27 \mathrm{MeV}$ and $35 \mathrm{MeV}$ and compare with the data from [14]. The ${ }^{6} \mathrm{Li}$ nucleus is treated within a twocluster model $(\alpha+d)$. The EBU contribution is obtained from the CDCC calculations. The $\alpha-d$ interaction is taken from [15]. The $d-{ }^{209} \mathrm{Bi}$ and 

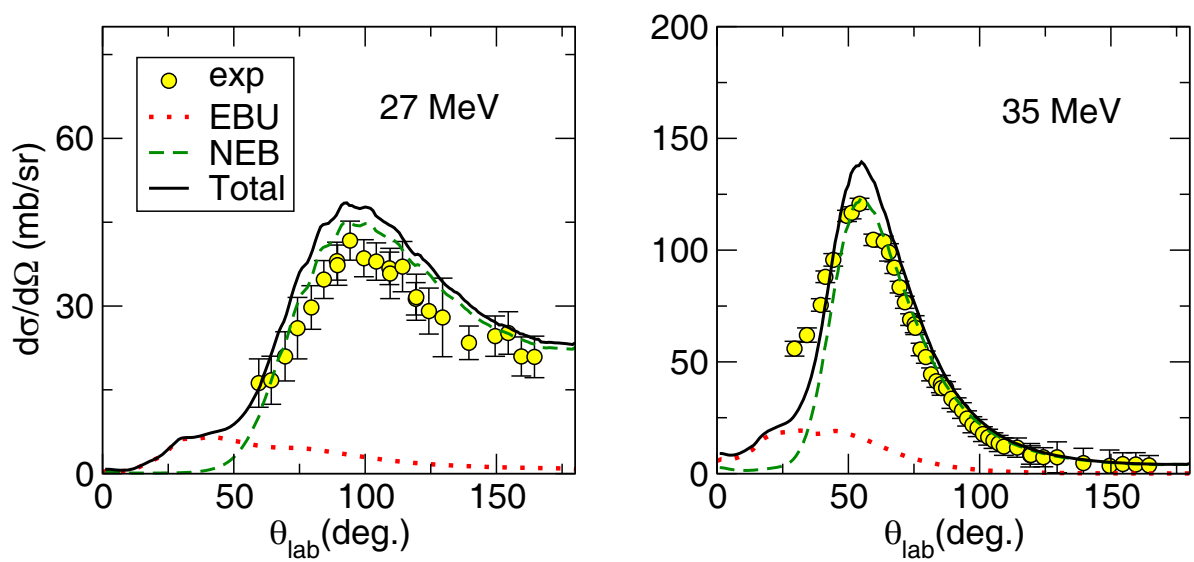

Figure 2: Experimental and calculated differential cross section angular distribution for the ${ }^{159} \mathrm{~Tb}\left({ }^{6} \mathrm{Li}, \alpha \mathrm{X}\right)$ reaction at energies of $27 \mathrm{MeV}$ (left panel) and $35 \mathrm{MeV}$ (right panel).

$\alpha-{ }^{159} \mathrm{~Tb}$ optical potentials are taken from Refs. [13] and [16], respectively. For the NEB calculation we adopt the same optical potential of $\alpha / d+{ }^{159} \mathrm{~Tb}$ as used in the CDCC calculations. For the ${ }^{6} \mathrm{Li}+{ }^{159} \mathrm{~Tb}$ optical potential we adopted the parametrization of Cook [17].

In Fig. 2, we compare the calculated and experimental angular distributions of $\alpha$ particles, for the two considered energies of ${ }^{6} \mathrm{Li}$. The dotted line is the calculation of EBU, which only contributes significantly at the very forward angles, whereas the dashed line is the result of NEB, which dominates the whole range of $\alpha$ production except the smallest angles. The summed EBU + NEB cross sections (thick solid lines) reproduce fairly well the shape and magnitude of the data.

\section{Conclusion}

In summary, we have investigated the problem of inclusive breakup in reactions induced by weakly bound nuclei. For the EBU part, we have considered the CDCC method, whereas for the NEB part, we revisited the model proposed by Ichimura, Austern and Vincent. We have performed calculations for ${ }^{118} \mathrm{Sn}(d, p X)$ and ${ }^{169} \mathrm{~Tb}\left({ }^{6} \mathrm{Li}, \alpha X\right)$, finding in both cases a good agreement with existing data and a dominance of the NEB contribution. Calculations for other systems are underway. 


\section{Acknowledgements}

This work has been partially supported by the Spanish Ministerio de Economía y Competitividad, under grant FIS2013-41994-P, by the Spanish Consolider-Ingenio 2010 Programme CPAN (CSD2007-00042) and by Junta de Andalucía (FQM160, P07-FQM-02894). J.L. is partially supported by a grant funded by the China Scholarship Council.

\section{References}

[1] J. Pampus, et al, Nucl. Phys. A311, 141 (1978)

[2] T. Udagawa, T. Tamura, Phys. Rev. C 24, 1348 (1981)

[3] N. Austern, C.M. Vincent, Phys. Rev. C 23(5), 1847 (1981)

[4] A. Kasano, M. Ichimura, Phys. Lett. 115B, 81 (1982)

[5] M. Ichimura, N. Austern, C.M. Vincent, Phys. Rev. C 32, 431 (1985)

[6] J. Lei, A. Moro, Phys. Rev. C submitted (2015)

[7] Y. Sokhotski, Saint Petersburg Doctor thesis (1873)

[8] N. Austern, et al, Phys. Rep. 154, 125 (1987)

[9] I.J. Thompson, Comp. Phys. Rep. 7, 167 (1988)

[10] N. Matsuoka, et al, Nucl.Phys. A345, 1 (1980)

[11] B.V. Carlson, R. Capote, M. Sin, (2015)

[12] A. Koning, J. Delaroche, Nucl. Phys. A 713(34), 231 (2003)

[13] Y. Han, Y. Shi, Q. Shen, Phys. Rev. C 74, 044615 (2006)

[14] M.K. Pradhan, et al, Phys. Rev. C 88, 064603 (2013)

[15] H. Nishioka, et al, Nucl. Phys. A 415, 230 (1984)

[16] J. Huizenga, G. Igo, Nuclear Physics 29, 462 (1962)

[17] J. Cook, Nucl. Phys. A 388, 153 (1982) 